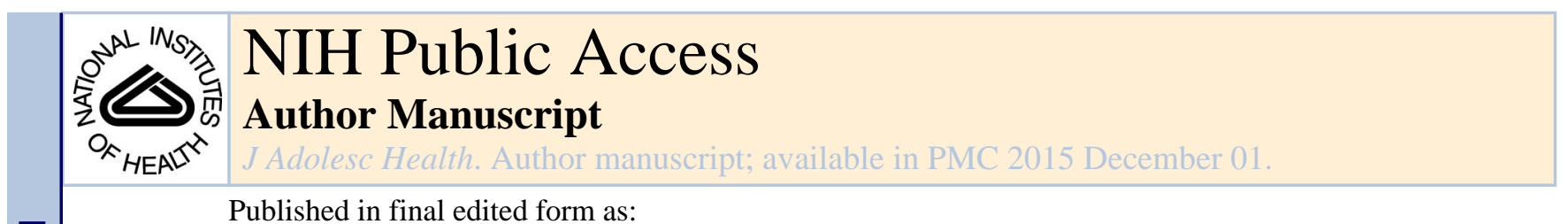

Published in final edited form as:

J Adolesc Health. 2014 December ; 55(6): 830-834. doi:10.1016/j.jadohealth.2014.07.006.

\title{
Gender Influences on Hepatitis C Incidence Among Street Youth in a Canadian Setting
}

\author{
Nitasha Puri, MD, CCFP1 ${ }^{1}$ Kora DeBeck, $\mathrm{PhD}^{1,2}$, Cindy Feng, $\mathrm{PhD}^{3}$, Thomas Kerr, $\mathrm{PhD}^{1,2}$, \\ Launette Rieb, MD, MSc, CCFP ${ }^{1,4}$, and Evan Wood, MD, PhD, ABIM, FRCPC ${ }^{1,5}$ \\ ${ }^{1}$ British Columbia Centre for Excellence in HIV/AIDS \\ ${ }^{2}$ School of Public Policy, Simon Fraser University \\ ${ }^{3}$ School of Public Health, University of Saskatchewan \\ ${ }^{4}$ Department of Family Medicine, University of British Columbia \\ ${ }^{5}$ Department of Medicine, University of British Columbia
}

\begin{abstract}
Purpose-Few studies have examined gender-based differences in the risk of hepatitis C (HCV) infection among street-involved youth. We compared rates of HCV infection among male and female street-involved youth in a Canadian setting.

Methods-The At-Risk Youth Study (ARYS) is a prospective cohort of drug-using, streetinvolved youth. Study recruitment and follow-up occurred in Vancouver, Canada, between September 2005 and November 2011. Eligible participants were illicit drug-using youth aged 1426 years at enrollment, recruited by street-based outreach. We evaluated rates of HCV antibody seroconversion, measured every six months during study follow-up, and used Cox proportional hazards regression to compare risk factors for $\mathrm{HCV}$ incidence between male and female street youth.
\end{abstract}

Results-Among $512 \mathrm{HCV}$-seronegative youth contributing 836 person-years of follow-up, 56 (10.9\%) seroconverted to HCV. Among female participants, the incidence density of HCV infection was 10.9 per 100 person-years and in males 5.1 per 100 person-years $(p=0.009)$. In multivariate analyses, female gender was independently associated with a higher rate of $\mathrm{HCV}$ seroconversion (Adjusted Hazard Ratio (AHR) $=2.01$; 95\% Confidence Interval [CI], 1.18 3.44). Risk factors were similar in gender stratified analyses and included injection heroin and injection crystal methamphetamine, although syringe sharing was only associated with HCV incidence among males.

(C) 2014 Society for Adolescent Medicine. Published by Elsevier Inc. All rights reserved.

Send correspondence to: Evan Wood, MD, PhD, ABIM, FRCPC, Director, Urban Health Research Initiative, B.C. Centre for Excellence in HIV/AIDS, University of British Columbia, St. Paul's Hospital, 608-1081 Burrard Street, Vancouver, B.C., V6Z 1Y6, Canada, Tel: (604) 806-9116, Fax: (604) 806-9044, urhi-ew@cfenet.ubc.ca.

Publisher's Disclaimer: This is a PDF file of an unedited manuscript that has been accepted for publication. As a service to our customers we are providing this early version of the manuscript. The manuscript will undergo copyediting, typesetting, and review of the resulting proof before it is published in its final citable form. Please note that during the production process errors may be discovered which could affect the content, and all legal disclaimers that apply to the journal pertain. 
Conclusions-Among street-involved youth in this setting, females had double the incidence of $\mathrm{HCV}$ seroconversion demonstrating the need for gender focused $\mathrm{HCV}$ prevention interventions for this population.

\section{Keywords}

Hepatitis C; street youth; gender; crystal methamphetamine; heroin

\section{INTRODUCTION}

Hepatitis C virus (HCV) infection is a growing public health issue (1-4). While the infection was originally associated with nosocomial transmission through the blood supply, recent trends demonstrate that the global burden of $\mathrm{HCV}$ infection is increasingly a result of illicit drug injection (2,5-8). For instance, in Canada, 70-80\% of HCV-infected individuals are people who inject drugs $(3,4)$.

Similarly, studies have shown that, in North America, HCV is highly prevalent among street-involved youth. In this population, $\mathrm{HCV}$ infection rates range from approximately $8 \%$ to $22 \%$ (9-12). Previously described risk factors associated with HCV among this population include specific injection practices, such as sharing syringes, as well as specific drug use patterns including longer duration of injecting, and using both heroin and cocaine by injection (13-16). In addition, in some gender-based analyses of the risks for HIV and/or $\mathrm{HCV}$ infection, studies have also shown females to be vulnerable to infection due to concurrent sexual and injection partnerships, trauma, sex work, and lack of control over where drugs are acquired and prepared (17-19).

In particular, the intersection between gender and vulnerability to $\mathrm{HCV}$ infection among street-involved youth is less understood. Although a street-involved cohort has not been reflected in the literature in detail, a few studies done with Aboriginal youth have suggested that young females are more vulnerable to HCV infection than males (20), while other studies have had more equivocal results (21). As such, the present study was conducted to assess the potential relationship between HCV incidence and gender among street-involved youth in a Canadian setting, as well as to explore if there were unique risk factors for $\mathrm{HCV}$ incidence according to gender in this setting.

\section{METHODS}

Data for this study were obtained from the At-Risk Youth Study (ARYS), which is a prospective cohort study of street-involved youth in Vancouver, Canada that began in 2005 and has been described in detail previously (5). The ARYS cohort's primary objective is to assess risk factors for initiation into injecting drug use. However, the study is positioned to examine a host of other secondary outcomes including risk for infectious diseases, and all participants receive HCV and HIV screening at baseline and semi-annually.

ARYS participants were recruited through snowball sampling and extensive street-based outreach methods. The street-based recruitment approach produced a sample of youth who spent extensive time on the street, a large proportion of whom were homeless. To be 
eligible, participants at recruitment must have been aged 14-26 years; used illicit drugs other than marijuana in the past 30 days; be 'street-involved,' defined as having been homeless in the past six months or recently having used a service for street-involved youth (e.g. housing or nutrition support) and had to provide written informed consent; and had to provide written informed consent. At enrollment and on a bi-annual basis, participants underwent $\mathrm{HCV}$ testing and completed a standardized interviewer-administered questionnaire that includes questions related to demographic information and drug use patterns. In addition, a study nurse examined participants for stigmata of drug injecting. The questionnaire and derived variables were defined based on earlier studies of street involved and adult drug users $(5,14)$. At each study visit, participants were provided with a stipend (\$20 CDN) for their time. The University of British Columbia's Research Ethics Board approved the study.

The study period for the current analysis was September 2005 to November 2011. The outcome of interest was HCV incidence and the primary explanatory variable was gender. Therefore, data from all participants who were $\mathrm{HCV}$-seronegative at enrollment and had completed at least one follow-up visit during the study period to assess for subsequent $\mathrm{HCV}$ seroconversion were eligible for inclusion in the present analyses. The date of HCV seroconversion was estimated as the midpoint between the first HCV seronegative test and the follow up visit with a confirmed positive HCV test. Participants were censored at the date of their last follow up visit or at the time of HCV infection, whichever came first.

To determine whether there was an independent association between HCV incidence and gender, we a priori selected a range of secondary explanatory variables that we hypothesized might also be associated with HCV incidence. These variables included age (per year older); ethnicity (Caucasian vs. other); education, defined as completed high school or greater, or is currently enrolled in school (yes vs. no); homelessness, defined as having no fixed address, sleeping on the street, couch surfing, or staying in a shelter or hostel (yes vs. no); injection heroin use (yes vs. no); injection cocaine use (yes vs. no); injection crystal methamphetamine use (yes vs. no); crack cocaine inhalation (yes vs. no); syringe sharing (yes vs. no); methadone maintenance use (yes vs. no); unsafe sex, defined as not always using condom or other barrier (dental dam, etc.) during vaginal, anal or oral sex (yes vs. no); and sex work, defined as received money or gifts or food or shelter or clothes or drugs in exchange for sex (yes vs. no). All drug use and behavioural variables refer to circumstances over the previous six months, and were treated as time-updated covariates on the basis of semi-annual follow-up data.

To assess the relationship between gender and HCV seroconversion, as a first step we presented the characteristics of the study sample, stratified by gender. We then calculated the cumulative incidence of HCV seroconversion for male and female participants over study follow-up using Kaplan-Meier methods. Survival curves were compared using the logrank test.

Using Cox proportional regression models, we then estimated the unadjusted relative hazard and $95 \%$ confidence intervals for factors associated with $\mathrm{HCV}$ incidence in the overall sample. To fit the multivariate Cox models, we used a backwards selection process 
previously described by Maldonado and Greenland (22) and Rothman and Greenland (23). Specifically, we began with all explanatory variables of interest in a full model. Using an automated procedure, we subsequently generated a series of confounding models by removing each secondary explanatory variable one at a time. For each of these models we assessed the relative change in the coefficient for our primary explanatory variable of interest (gender). The secondary explanatory variable of interest that resulted in the smallest absolute relative change in the coefficient for gender was then removed. Secondary variables continued to be removed through this process until the smallest relative change in the coefficient for the effect of gender on HCV seroconversion exceeded 5\% of the value of the coefficient. Remaining variables were considered confounders and were included in the final multivariate analysis.

As a sub-analysis, we then assessed risk factors associated with HCV seroconversion among male and female youth, separately in stratified models. The same variables of interest were considered and two separate multivariate Cox regressions were constructed. Model selection was done based on the Akaike Information Criterion (AIC) with the best subset selection procedure. This provided a computationally efficient method to screen all possible combinations of candidate variables and identify the model with the best overall fit as indicated by the lowest AIC value (24). All statistical analyses were performed using SAS software version 9.2 (SAS, Cary, NC, USA). All tests of significance were two-sided, and a $p$-value of 0.05 or less was selected for defining statistical significance.

\section{RESULTS}

From September 2005 to November 2011, 940 youth were recruited into the ARYS cohort and completed baseline HCV antibody testing. The baseline prevalence of HCV seropositivity was $10.6 \%$. Among the 840 (89.4\%) individuals who were HCV seronegative at baseline, 512 (60.9\%) completed at least one study follow-up and HCV antibody test, and were therefore eligible for the present study.

In comparison to the 512 youth who represented the eligible study population, the 428 youth who were ineligible for the present study because they did not have a follow up HCV antibody test were more likely to be Caucasian $(p=0.008)$ but did not differ based on having completed high school or currently being enrolled in school $(p=0.697)$, being older $(p=0.841)$, or gender $(p=0.496)$.

Among the sample of 512 participants included in the study, 151 (29.5\%) were female, and the median age was 22 (Interquartile range $18.8-23.0$ ). As shown in Table 1, male and female participants differed in four demographic characteristics (where $p<0.05$ ); males were older than females and had a higher rate of homelessness, while females had a higher rate of high school completion and involvement in sex work.

Over the course of study follow-up, $56(10.9 \%) \mathrm{HCV}$ seroconversion events were observed for an incidence density of 6.5 per 100 person years (95\% Confidence Interval [CI] $7.98-$ 12.58). Among female participants, the incidence density of $\mathrm{HCV}$ infection was 10.9 per 100 person-years and in males it was 5.1 per 100 person-years $(p=0.009)$. The Kaplan- 
Meier estimates of the cumulative incidence of $\mathrm{HCV}$ seroconversion stratified by gender are shown in Figure 1. As shown here, female participants were at higher risk of HCV seroconversion compared with male participants $(\log -\operatorname{rank} p=0.005)$. The cumulative incidence of HCV seroconversion among female participants reached $45.6 \%$ over the 3-year follow-up period compared to $16.6 \%$ among male participants.

Table 2 shows the unadjusted and adjusted relative hazard of HCV incidence. In univariate Cox regression analysis, gender was significantly associated with HCV seroconversion [Hazard Ratio $(\mathrm{HR})=2.10,95 \%$ CI:1.24-3.56]. In multivariate Cox regression analyses, gender remained significant [Adjusted Hazard Ratio $($ AHR $)=2.01,95 \%$ CI: $1.10-3.44$ ], as well as crystal methamphetamine injection [AHR $=3.38,95 \%$ CI: $1.85-6.19]$ and heroin injection $[\mathrm{AHR}=5.45,95 \% \mathrm{CI}: 2.91-10.21]$. Table 3 shows the multivariate analysis for risk factors associated with HCV seroconversion, stratified by gender. Among male streetinvolved youth, injection heroin use [AHR $=4.94,95 \%$ CI: $2.19-11.12$ ], injection crystal methamphetamine use [AHR $=3.06,95 \% \mathrm{CI}: 1.33-7.06]$ and history of sharing needles [AHR $=4.38,95 \%$ CI: $1.82-10.55]$ were positively associated with HCV seroconversion. Among female youth, injection heroin use $[\mathrm{AHR}=4.88,95 \% \mathrm{CI}$ : 2.04-11.68] and injection crystal methamphetamine use $[\mathrm{AHR}=3.93,95 \%$ CI: $1.66-9.33]$ were positively associated with subsequent $\mathrm{HCV}$ seroconversion.

\section{DISCUSSION}

Among street-involved youth in our study, we observed that HCV incidence was significantly elevated among females versus males and that risk factors for HCV incidence were generally similar. Specifically, for both male and female streetinvolved youth, risk factors for HCV seroconversion included injection crystal methamphetamine and injection heroin use. In addition, a history of sharing needles was a risk factor for seroconversion to HCV for males but not for females in our study.

A number of studies have examined injection drug related harms and found that in addition to $\mathrm{HCV}$, injection drug use is associated with an elevated risk for HIV, nonfatal overdoses (both heroin and crystal methamphetamine)(10), as well as soft tissue infections and infective endocarditis (25). Gender-based studies have shown that rates of initiation to injection drug use are often similar among both genders, although risk factors are different $(25,26)$. The incidence of HIV has previously been shown to be slightly higher in females who use injection drugs according to some studies $(20,26)$.

Interestingly, in Canada, 2010 surveillance reports have suggested that HCV is more prevalent among males than females in the general population, despite a narrowing gap $(3,11)$. New cases of HCV in males are reportedly greatest between ages 40 and 59, whereas in females incidence is highest among youth aged 15-24 $(2,11)$. Our findings suggest that the overall gender gap in HCV may be closing with higher incidence rates among female street involved youth. In fact, these data are consistent with data from a study of Aboriginal youth in British Columbia which found that Aboriginal female youth were also more likely to seroconvert compared to males (20). Together, these studies demonstrate that young female street-involved drug users may be particularly vulnerable to $\mathrm{HCV}$ infection. In 
addition, in this particular study, the gap between incidence in males and females is higher than most other studies.

Although our results are consistent with some earlier literature with respect to HCV incidence in youth and females, our risk factor analysis differs from other studies, an area which merits further research. Previous analyses have shown that females are more vulnerable due to sharing syringes, being involved in sex work, having the same partner concurrently for injection and sex, reliance on friends and partners for drugs, and a lack of control over drug acquisition, preparation, and injection $(15,17,18,20)$. Interestingly, our study did not show any risk from sex work or sharing syringes. Similarly, other studies have shown that injection heroin and cocaine, as well as longer duration of injection, are risk factors for HCV seroconversion among females (13-16). Our study examined crystal methamphetamine injection, a variable that is not well described in other studies, and found it to be statistically significant. This particular finding requires further analysis, including whether there is a greater correlation between female gender and crystal methamphetamine use, and what unique practices may be associated with crystal methamphetamine use that may put both male and female youth at greater risk of HCV infection.

There are several limitations to our study. First, as with other studies of street-involved youth, the ARYS cohort is not a random sample, and therefore these findings may not generalize to other street youth populations. Second, although the primary outcome (HCV incidence) was based on a biological measure, this study also used self-reported behavioural information which is susceptible to recall bias and socially desirable responding (5). It was interesting in the present study that syringe sharing was not associated with HCV incidence among female participants. It is possible that despite our efforts to engage in a nonjudgmental way, female participants underreported those activities perceived as socially unacceptable, such as syringe sharing. Nevertheless, our study's main endpoint was based on a biological measure indicating that $\mathrm{HCV}$ incidence is higher in female street youth in this setting.

In summary, the present study found that the incidence of new HCV infection is over twice as high among female street-involved youth compared to males, and that risk factors for seroconversion are largely similar between both genders. The two-fold higher incidence of $\mathrm{HCV}$ among females despite multivariate adjustment suggests that HCV prevention efforts may benefit from considering and further studying the unique characteristics and vulnerabilities of female street-involved youth. Here, qualitative research may be helpful to further elucidate these specific risks. For both genders, targeting interventions to specific risk factors of injection drug use, notably crystal methamphetamine and heroin, is suggested. Given the serious health harms and vulnerabilities experienced by this population, evidencebased and gender specific interventions involving scaling up addiction treatment and $\mathrm{HCV}$ prevention strategies are urgently needed.

\section{Acknowledgments}

Funding: The authors thank the study participants for their contribution to the research, as well as current and past researchers and staff. We would specifically like to thank Cody Callon, Jennifer Matthews, Deborah Graham, Peter Vann, Steve Kain, Tricia Collingham, Kristie Starr, and Carmen Rock for their research and administrative 
assistance. The study was supported by the US National Institutes of Health (R01DA028532) and the Canadian Institutes of Health Research (MOP-102742). This research was undertaken, in part, thanks to funding from the Canada Research Chairs program through a Tier 1 Canada Research Chair in Inner City Medicine which supports Dr. Evan Wood. Dr. Kora DeBeck is supported by a MSFHR/St. Paul's Hospital-Providence Health Care Career Scholar Award. Funding sources had no further role in study design; or in the decision to submit the paper for publication.

\section{REFERENCES}

1. Klein MB, Rollet KC, Saeed S, et al. HIV and hepatitis C virus coinfection in Canada: challenges and opportunities for reducing preventable morbidity and mortality. HIV Med. 2013; 14:10-20. [PubMed: 22639840]

2. Public Health Agency of Canada. Hepatitis C in Canada: 2005-2010 Surveillance Report. Available online: http://www.phac-aspc.gc.ca/sti-its-surv-epi/hepc/surv-eng.php.

3. L C. The epidemiology of hepatitis C in Canada. Available at: http://www.catie.ca/en/fact-sheets/ epidemiology/epidemiology-hepatitis-c-canada.

4. Wong T, Lee SS. Hepatitis C: a review for primary care physicians. Cmaj. 2006; 174:649-659. [PubMed: 16505462]

5. Wood E, Stoltz JA, Montaner JS, et al. Evaluating methamphetamine use and risks of injection initiation among street youth: the ARYS study. Harm Reduct J. 2006; 3:18. [PubMed: 16723029]

6. Centre for Disease Control and Prevention. HIV Transmission. 2010 Available online: http:// www.bccdc.ca/NR/rdonlyres/2035512C-DBEC-495B-A332-C410EE9520C7/0/ CPS_Report_STI_HIV_2010_annual_report_FINAL_20111122.pdf.

7. Centre for Disease Control and Prevention. [Accessed January 7 2014] Recommendations for Prevention and Control of Hepatitis C Virus (HCV) Infection and HCV-Related Chronic Disease. 1998. Available online: http://www.cdc.gov/mmwr/PDF/rr/rr4719.PDF.

8. Wright NM, Tompkins CN. A review of the evidence for the effectiveness of primary prevention interventions for hepatitis $\mathrm{C}$ among injecting drug users. Harm Reduct J. 2006; 3:27. [PubMed: 16956393]

9. Miller CL, Kerr T, Fischer B, et al. Methamphetamine injection independently predicts hepatitis C infection among street-involved youth in a Canadian setting. J Adolesc Health. 2009; 44:302-304. [PubMed: 19237118]

10. Kerr T, Marshall BD, Miller C, et al. Injection drug use among street-involved youth in a Canadian setting. BMC Public Health. 2009; 9:171. [PubMed: 19493353]

11. Public Health Agency of Canada. Population-specific Status Report: HIV/AIDS and other sexually transmitted and blood borne infections among youth in Canada: Public Health Agency of Canada. 2014 Available online: http://librarypdf.catie.ca/pdf/ATI-20000s/26485.pdf.

12. Roy E, Haley N, Leclerc P, et al. Risk factors for hepatitis $\mathrm{C}$ virus infection among street youths. CMAJ. 2001; 165:557-560. [PubMed: 11563207]

13. Spittal PM, Pearce ME, Chavoshi N, et al. The Cedar Project: high incidence of HCV infections in a longitudinal study of young Aboriginal people who use drugs in two Canadian cities. BMC Public Health. 2012; 12:632. [PubMed: 22877418]

14. Roy E, Arruda N, Leclerc P, et al. Injection of drug residue as a potential risk factor for $\mathrm{HCV}$ acquisition among Montreal young injection drug users. Drug Alcohol Depend. 2012; 126:246250. [PubMed: 22699096]

15. Hahn JA, Page-Shafer K, Lum PJ, et al. Hepatitis C virus seroconversion among young injection drug users: relationships and risks. J Infect Dis. 2002; 186:1558-1564. [PubMed: 12447730]

16. Miller CL, Johnston C, Spittal PM, et al. Opportunities for prevention: hepatitis C prevalence and incidence in a cohort of young injection drug users. Hepatology. 2002; 36:737-742. [PubMed: 12198668]

17. Evans JL, Hahn JA, Page-Shafer K, et al. Gender differences in sexual and injection risk behavior among active young injection drug users in San Francisco (the UFO Study). J Urban Health. 2003; 80:137-146. [PubMed: 12612103] 
18. Wagner KD, Bloom JJ, Hathazi SD, et al. Control over drug acquisition, preparation and injection: Implications for HIV and HCV risk among young female injection drug users. ISRN Addict. 2013:2013.

19. Mehrabadi A, Craib KJ, Patterson K, et al. The Cedar Project: a comparison of HIV-related vulnerabilities amongst young Aboriginal women surviving drug use and sex work in two Canadian cities. Int J Drug Policy. 2008; 19:159-168. [PubMed: 17870461]

20. Mehrabadi A, Paterson K, Pearce M, et al. Gender differences in HIV and hepatitis C related vulnerabilities among aboriginal young people who use street drugs in two Canadian cities. Women Health. 2008; 48:235-260. [PubMed: 19191041]

21. Hagan H, Des Jarlais DC, Stern R, et al. HCV synthesis project: preliminary analyses of HCV prevalence in relation to age and duration of injection. Int J Drug Policy. 2007; 18:341-351. [PubMed: 17854721]

22. Maldonado G, Greenland S. Simulation study of confounder-selection strategies. Am J Epidemiol. 1993; 138:923-936. [PubMed: 8256780]

23. Rothman, KGS. Modern Epidemiology. Philadelphia, Pennsylvania: Lippincott; 1998.

24. Shtetland, ESCE.; Barton, MB. SAS Institute, SUGI '26 Proceedings edition. Cary, NC: 2001. The perils of stepwise logistic regression and how to escape them using information criteria and the output delivery system; p. 222-226.

25. Ahamad K, Debeck K, Feng C, et al. Gender influences on initiation of injecting drug use. Am J Drug Alcohol Abuse. 2014; 40:151-156. [PubMed: 24405226]

26. Doherty MC, Garfein RS, Monterroso E, et al. Gender differences in the initiation of injection drug use among young adults. J Urban Health. 2000; 77:396-414. [PubMed: 10976613] 


\section{IMPLICATIONS AND CONTRIBUTION STATEMENT}

Among street involved youth in this setting, the incidence of $\mathrm{HCV}$ seroconversion was twice as high among female youth. These findings underscore the need for evidencebased and gender specific HCV prevention and addiction treatment interventions targeting vulnerable street-involved youth. 


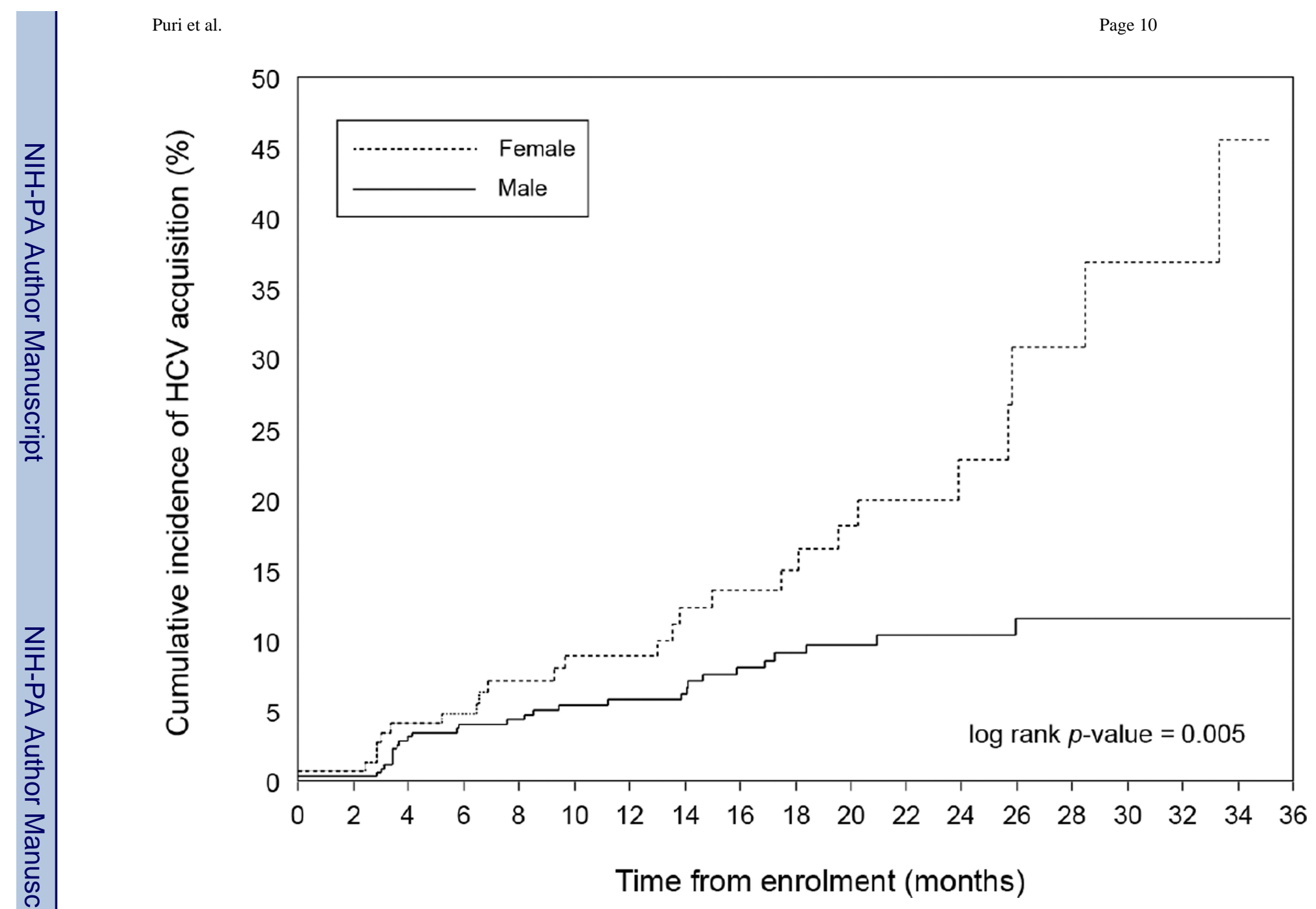

Figure 1.

Cumulative incidence of HCV among street-involved youth stratified by gender Sep 2005 Nov $2011(n=512)$. 


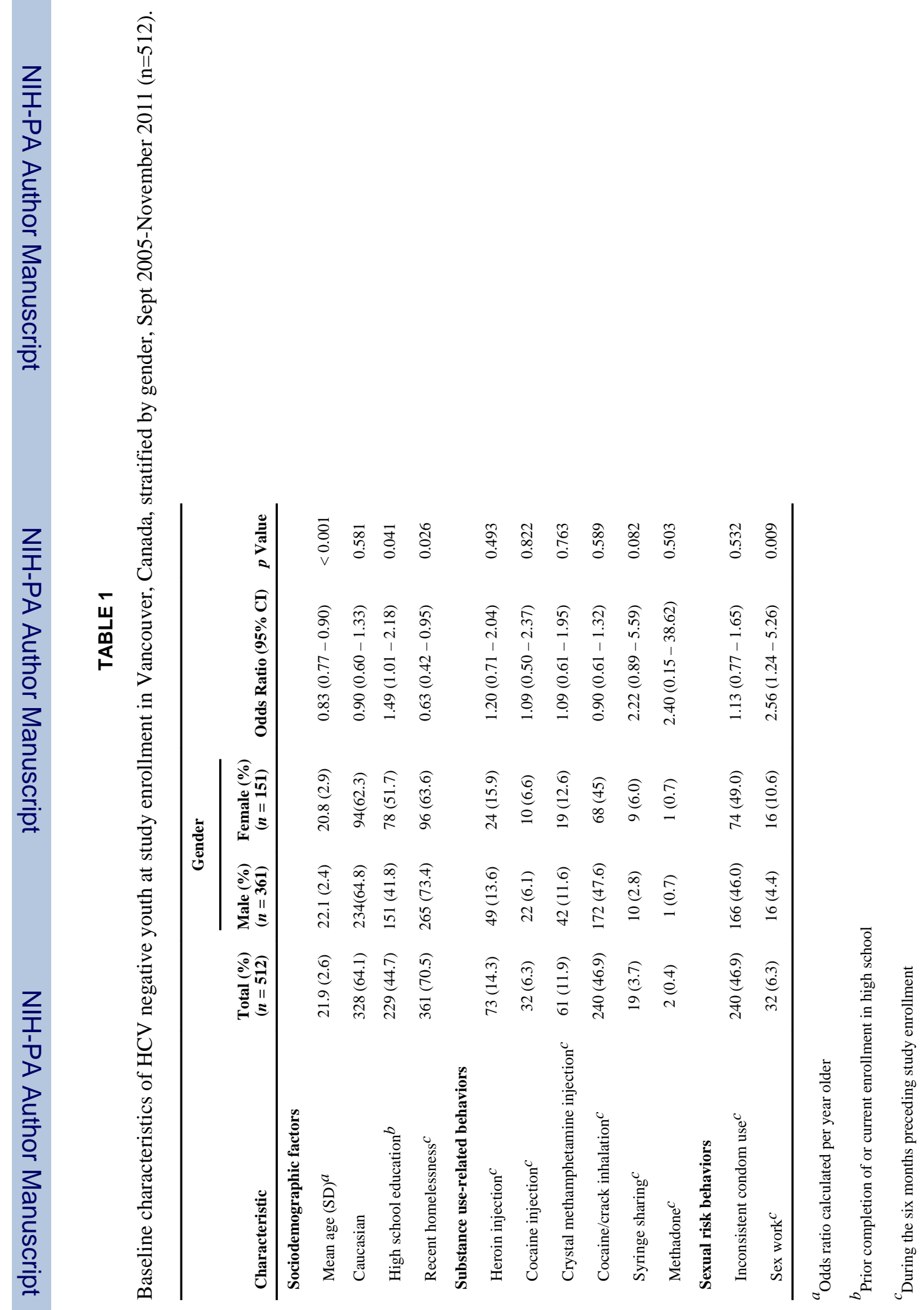




\section{Table 2}

Bivariate and multivariate cox regression analysis of factors associated with HCV incidence among streetinvolved youth in Vancouver $(\mathrm{n}=512)$.

\begin{tabular}{|c|c|c|c|c|}
\hline \multirow[b]{2}{*}{ Characteristic } & \multicolumn{2}{|c|}{ Unadjusted } & \multicolumn{2}{|c|}{ Adjusted } \\
\hline & $\begin{array}{l}\text { Hazard Ratio } \\
\text { (95\% CI) }\end{array}$ & $p$ - value & $\begin{array}{c}\text { Hazard Ratio } \\
(\mathbf{9 5 \%} \text { CI })\end{array}$ & $p$ - value \\
\hline \multicolumn{5}{|l|}{ Gender } \\
\hline (female vs. male) & $2.10(1.24-3.56)$ & 0.006 & $2.01(1.18-3.44)$ & 0.011 \\
\hline \multicolumn{5}{|l|}{ Age } \\
\hline (per year older) & $0.96(0.87-1.06)$ & 0.373 & & \\
\hline \multicolumn{5}{|l|}{ Caucasian } \\
\hline (yes vs. no) & $1.12(0.65-1.93)$ & 0.697 & & \\
\hline \multicolumn{5}{|l|}{ Sex work involved ${ }^{\dagger}$} \\
\hline (yes vs. no) & $0.91(0.28-2.90)$ & 0.869 & & \\
\hline \multicolumn{5}{|l|}{ Unsafe $\operatorname{sex}^{\dagger}$} \\
\hline (yes vs. no) & $0.90(0.52-1.55)$ & 0.703 & & \\
\hline \multicolumn{5}{|l|}{ Homeless ${ }^{\dagger}$} \\
\hline (yes vs. no) & $1.23(0.72-2.09)$ & 0.451 & & \\
\hline \multicolumn{5}{|l|}{ Education* } \\
\hline (yes vs. no) & $0.56(0.33-0.96)$ & 0.036 & & \\
\hline \multicolumn{5}{|c|}{ Crystal meth injection ${ }^{\dagger}$} \\
\hline (yes vs. no) & $7.39(4.36-12.52)$ & $<0.001$ & $3.38(1.85-6.19)$ & $<0.001$ \\
\hline \multicolumn{5}{|l|}{ Cocaine injection ${ }^{\dagger}$} \\
\hline (yes vs. no) & $5.69(3.18-10.20)$ & $<0.001$ & & \\
\hline \multicolumn{5}{|l|}{ Heroin injection ${ }^{\dagger}$} \\
\hline (yes vs. no) & $9.89(5.72-17.10)$ & $<0.001$ & $5.45(2.91-10.21)$ & $<0.001$ \\
\hline \multicolumn{5}{|c|}{ Crack cocaine smoking ${ }^{\dagger}$} \\
\hline (yes vs. no) & $0.48(0.23-1.03)$ & 0.059 & & \\
\hline \multicolumn{5}{|l|}{ Syringe sharing ${ }^{\dagger}$} \\
\hline (yes vs. no) & $7.69(3.93-15.04)$ & $<0.001$ & & \\
\hline \multicolumn{5}{|l|}{ methadone use ${ }^{\dagger}$} \\
\hline (yes vs. no) & $12.05(1.64-88.44)$ & 0.014 & & \\
\hline
\end{tabular}




\section{TABLE 3}

Multivariate cox regressions for factors associated with HCV incidence among female and male streetinvolved youth in Vancouver, Canada, September 2005- November $2011(n=512)$.

\begin{tabular}{|c|c|c|}
\hline Characteristic & $\begin{array}{c}\text { Adjusted HR }{ }^{a} \\
\quad\left(95 \% \mathbf{C I}^{b}\right)\end{array}$ & $p$ Value \\
\hline \multicolumn{3}{|l|}{ Model I: Male Gender } \\
\hline Injection heroin use ${ }^{c}$ (yes vs no) & $4.94(2.19-11.12)$ & $<0.001$ \\
\hline Injection crystal meth use ${ }^{c}$ (yes vs no) & $3.06(1.33-7.06)$ & 0.008 \\
\hline History of sharing needles ${ }^{c}$ (yes vs no) & $4.38(1.82-10.55)$ & 0.001 \\
\hline \multicolumn{3}{|l|}{ Model II: Female Gender } \\
\hline Injection heroin use ${ }^{c}$ (yes vs no) & $4.88(2.04-11.68)$ & $<0.001$ \\
\hline Injection crystal meth use ${ }^{c}$ (yes vs no) & $3.93(1.66-9.33)$ & 0.002 \\
\hline
\end{tabular}

Notes:

${ }^{a} \mathrm{HR}=$ Hazard Ratio;

${ }^{b} \mathrm{CI}=$ Confidence Interval;

${ }^{c}$ Denotes behavior in the preceding six months 